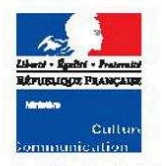

Secrétariat général

Service de la

coordination des

politiques culturelles

et de l'innovation

Département

des études,

de la prospective

et des statistiques

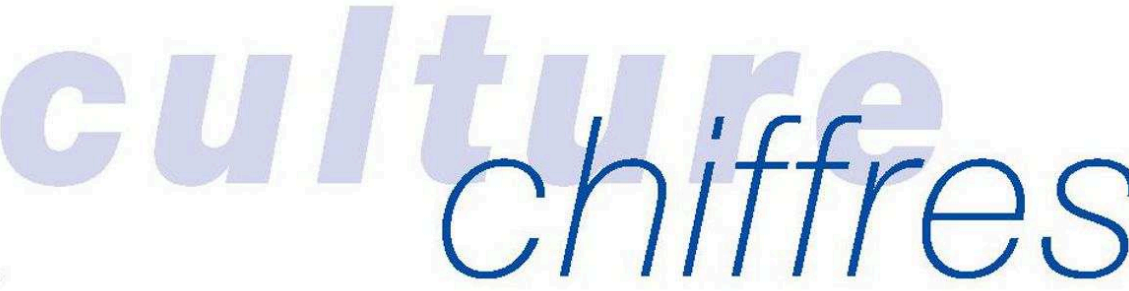

82, rue Saint-Honoré, 75033 Paris cedex 0

중 0140157917 -圂 0140157999

\title{
Repères économiques des secteurs culturels marchands en 2010
}

Valérie DERoIN*

\author{
Economic overview of the cultural \\ market-oriented sectors in $\mathbf{2 0 1 0}$
}

Décrire l'organisation économique des activités culturelles suppose d'intégrer et d'adopter des outils de mesure communs à des activités par ailleurs très diverses. Les chaînes économiques de l'ensemble des secteurs culturels marchands sont constituées de filières différentes qu’il reste malaisé de comparer : la production de spectacles et la production de livres diffèrent par le capital, les outils et les investissements nécessaires qu'elles exigent; l'activité de distribution de produits (stocks, charges financières, ventes, etc.) est difficilement comparable à celle de la création ou à celle de l'enseignement, etc. De plus, la spécificité de modèles d'affaires ou économiques plus ou moins dépendants d'un vol ume important de mesures de soutien public s'appréhende mal à travers l'analyse de variables comptables et financières utilisées dans les enquêtes économiques nationales ; enfin, l'offre professionnelle cohabite avec l'offre amateur dans bien des secteurs.

Pour autant, il est possible de dresser un panorama global des principales caractéristiques économiques du champ de la culture en 2010: ce champ, fondé sur des outils de mesure statistique et délimité à 29 activités, est constitué des secteurs culturels marchands identifiables par la nomenclature statistique d'activités et comparables aux autres secteurs de l'économie française.

\section{DÉFINITION DU CHAMP}

L'analyse du champ de la culture renvoie à un corpus d'activités plus ou moins large, et peut produire des résultats d'amplitude variable. De fait, le secteur culturel n'a pas d'existence économique en tant que regroupement de familles de produits ou services culturels qui permettrait de border le champ. La palette des activités culturelles tend elle-même à s'élargir, en particulier sous l'effet de développements technologiques qui apportent de nouveaux produits culturels, de nouveaux acteurs, de nouveaux réseaux d'activités.

Pour autant, l'Union européenne s'est dotée d'un cadre statistique de la culture, défini conjointement par les États membres au sein du réseau d'experts européens Essnet
Culture : ce cadre statistique est utilisé pour représenter le champ d'étude des secteurs culturels'. Les États membres ont ainsi retenu des critères communs pour structurer un système d'observation des activités culturelles en Europe. Les activités culturelles représentent des activités qui reposent sur des valeurs culturelles et/ou une expression artistique ; elles placent la création au centre du système. Les activités publicitaires, par exemple, sont reconnues comme activités culturelles par leurs activités de création de scénarios.

Plus précisément, les activités culturelles, artistiques ou créatives définies par le champ Essnet de la culture s'insèrent dans dix grands domaines culturels:

-le patrimoine des musées, des monuments historiques et de l'archéologie ;

* Chargée d'analyses statistiques.

1. Voir Valérie DEROIN, Approche statistique européenne de la culture, Paris, Ministère de la Culture et de la Communication, DEPS, coll. «Culture études», 2011-8, décembre 2011. 


\section{Repères économiques des secteurs culturels marchands en 2010}

Economic overview of the cultural market-oriented sectors in 2010

\section{Valérie Deroin}

Éditeur : Département des études, de la prospective et des statistiques

Lieu d'édition : Paris

Année d'édition : 2012

Date de mise en ligne : 21 septembre 2015

Collection : Culture chiffres

ISBN électronique : 9782111398610

\section{Sbooks}

http://books.openedition.org

\section{Édition imprimée}

Date de publication : 1 septembre 2012

Nombre de pages : 12

\section{Référence électronique}

DEROIN, Valérie. Repères économiques des secteurs culturels marchands en 2010. Nouvelle édition [en ligne]. Paris : Département des études, de la prospective et des statistiques, 2012 (généré le 25 avril 2021). Disponible sur Internet : <http://books.openedition.org/deps/508>. ISBN : 9782111398610. 

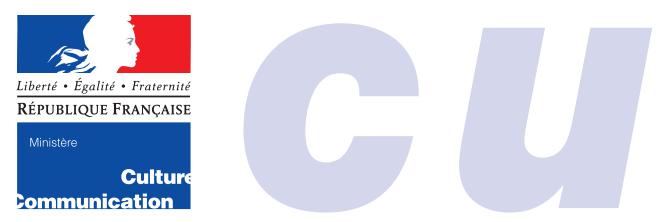

Secrétariat général

Service de la

coordination des

politiques culturelles

et de l'innovation

Département

des études,

de la prospective

et des statistiques

\title{
Repères économiques des secteurs culturels marchands en $\mathbf{2 0 1 0}$
}

\author{
Valérie DEROIN*
}

\section{Economic overview of the cultural market-oriented sectors in 2010}

Décrire l'organisation économique des activités culturelles suppose d'intégrer et d'adopter des outils de mesure communs à des activités par ailleurs très diverses. Les chaînes économiques de l'ensemble des secteurs culturels marchands sont constituées de filières différentes qu'il reste malaisé de comparer : la production de spectacles et la production de livres diffèrent par le capital, les outils et les investissements nécessaires qu'elles exigent ; l'activité de distribution de produits (stocks, charges financières, ventes, etc.) est difficilement comparable à celle de la création ou à celle de l'enseignement, etc. De plus, la spécificité de modèles d'affaires ou économiques plus ou moins dépendants d'un volume important de mesures de soutien public s'appréhende mal à travers l'analyse de variables comptables et financières utilisées dans les enquêtes économiques nationales; enfin, l'offre professionnelle cohabite avec l'offre amateur dans bien des secteurs.

Pour autant, il est possible de dresser un panorama global des principales caractéristiques économiques du champ de la culture en 2010 : ce champ, fondé sur des outils de mesure statistique et délimité à 29 activités, est constitué des secteurs culturels marchands identifiables par la nomenclature statistique d'activités et comparables aux autres secteurs de l'économie française.

\section{DÉFINITION DU CHAMP}

L'analyse du champ de la culture renvoie à un corpus d'activités plus ou moins large, et peut produire des résultats d'amplitude variable. De fait, le secteur culturel n'a pas d'existence économique en tant que regroupement de familles de produits ou services culturels qui permettrait de border le champ. La palette des activités culturelles tend elle-même à s'élargir, en particulier sous l'effet de développements technologiques qui apportent de nouveaux produits culturels, de nouveaux acteurs, de nouveaux réseaux d'activités.

Pour autant, l'Union européenne s'est dotée d'un cadre statistique de la culture, défini conjointement par les États membres au sein du réseau d'experts européens Essnet
Culture : ce cadre statistique est utilisé pour représenter le champ d'étude des secteurs culturels ${ }^{1}$. Les États membres ont ainsi retenu des critères communs pour structurer un système d'observation des activités culturelles en Europe. Les activités culturelles représentent des activités qui reposent sur des valeurs culturelles et/ou une expression artistique; elles placent la création au centre du système. Les activités publicitaires, par exemple, sont reconnues comme activités culturelles par leurs activités de création de scénarios.

Plus précisément, les activités culturelles, artistiques ou créatives définies par le champ Essnet de la culture s'insèrent dans dix grands domaines culturels :

- le patrimoine des musées, des monuments historiques et de l'archéologie ; 
- les archives ;

- les bibliothèques ;

- le livre et la presse ;

- les arts visuels (arts plastiques, photographie, design);

- le spectacle vivant;

- l'audiovisuel et le multimédia ;

- l'architecture ;

- la publicité ;

- l'artisanat d'art.

Les activités culturelles elles-mêmes résultent du croisement de ces domaines culturels avec des fonctions économiques : celles de création, de production et d'édition, de diffusion et de commercialisation, de conservation, de formation et d'administration et de management culturels. La construction de ce champ de la culture en Europe permet ainsi de disposer d'un ensemble cohérent et reconnu d'activités culturelles, prérequis nécessaire à la production de données européennes comparables et à l'analyse des secteurs économiques de la culture. Ces dix domaines culturels et ces six fonctions économiques sont utilisés pour la présente analyse des secteurs culturels marchands en France (voir encadré méthodologique).

\section{EN 2010, LA PART DES SECTEURS CULTURELS DANS L'ÉCONOMIE MARCHANDE EST D'ENVIRON $5 \%$ EN NOMBRE D'UNITÉS LÉGALES}

\section{ET DE PRÈS DE $3 \%$ \\ EN VALEUR AJOUTÉE}

Avec environ 157000 unités légales ${ }^{2}$ en 2010, la culture et ses différents secteurs d'activités représentent $4,7 \%$ de l'ensemble des secteurs marchands, hors agriculture. Ces unités réalisent un chiffre d'affaires de 71 milliards d'euros, soit $2 \%$ de l'économie marchande non agricole, et emploient près de 281000 salariés en équivalent temps plein, soit $2,3 \%$ des emplois marchands non agricoles (tableau 1). Les secteurs culturels apportent 29 milliards de valeur ajoutée à la production nationale, soit près de $3 \%$ de la valeur ajoutée totale des secteurs marchands non agricoles.

En rapportant la valeur ajoutée au chiffre d'affaires, soit la capacité de l'entreprise à générer de la richesse pour elle-même (autofinancement), pour ses salariés, pour l'État (impôts et taxes) et pour les banques (charges financières), on observe un taux moyen des secteurs culturels de $41 \%$, qui est supérieur au taux moyen de l'ensemble des secteurs marchands (29\%) mais légèrement inférieur au taux global moyen des services (information et communication : $45 \%$, activités spécialisées, scientifiques et techniques et activités de services administratifs et de soutien : $48 \%$ ).

L'excédent brut d'exploitation (EBE) correspond à 8,3 milliards d'euros pour le champ culturel, soit $3 \%$ de l'ensemble des secteurs marchands. Comme les entreprises n'ont pas toutes le même mode de financement de leur activité, l'EBE, qui mesure la performance des activités d'exploitation, se révèle utile pour comparer leurs ressources après paiement des impôts, taxes et charges de personnel. L'EBE révèle de fortes disparités entre secteurs culturels marchands: les éditions de chaînes généralistes, l'architecture et la production de films pour la télévision (EBE entre 1,1 et 1,6 milliard d'euros) se démarquent nettement, par exemple, de la gestion des salles de spectacles, la gestion des monuments et des musées (EBE inférieur à 27 millions d'euros pour chacune de ces activités).

\section{Caractéristiques géNérales SELON LES SECTEURS CULTURELS}

\section{Architecture et arts visuels réunissent plus de la moitié du nombre d'unités}

En 2010, les activités d'architecture sont prépondérantes en nombre avec 31646 unités (tableau 2), soit $20 \%$ des secteurs culturels hors patrimoine (musées, monuments, archives, bibliothèques). Elles sont suivies des arts visuels, notamment le design qui compte 20024 unités (13\% des secteurs culturels) et la création artistique des écrivains, compositeurs de musique et journalistes indépendants avec 13819 unités (9\%). Parmi les activités de spectacle vivant, celles des artistes du spectacle vivant, de l'organisation et la production de spectacles sont réalisées par quelque dix milliers d'unités (11 097).

L'audiovisuel et le multimédia, dans une conception élargie depuis la production jusqu'à l'édition et la distribution, couvrent $12 \%$ des unités des secteurs culturels avec 19495 unités : on observe cependant une grande disparité de ces secteurs avec, d'un côté, l'édition de chaînes généralistes assurée par seulement 23 unités légales et, de l'autre, la postproduction audiovisuelle qui occupe 3913 unités.

\section{Architecture, édition de chaînes généralistes et publicité sont les principaux contributeurs économiques du champ culturel}

Premières en nombre d'unités, les activités d'architecture sont également les principales contributrices à l'ensemble du chiffre d'affaires et de la valeur ajoutée du champ culturel (14\% de la valeur ajoutée de l'ensemble et $10 \%$ du chiffre d'affaires). Ensuite, l'édition de chaînes généralistes $(11 \%$ de la valeur ajoutée de l'ensemble ainsi que $11 \%$ du chiffre d'affaires) et toutes les activités de publicité culturelles et non culturelles ( $16 \%$ de la valeur ajoutée de l'ensemble et $18 \%$ du chiffre d'affaires) contribuent pour plus de $10 \%$ à la valeur ajoutée (VA) et au chiffre

2. Personnes physiques et personnes morales, y compris les auto-entrepreneurs. Voir rubrique « Définitions », p. 10. 


\begin{tabular}{|c|c|c|c|}
\hline Indicateur & $\begin{array}{l}\text { Secteurs culturels } \\
\text { marchands }\end{array}$ & $\begin{array}{l}\text { Tous secteurs } \\
\text { marchands }\end{array}$ & $\begin{array}{c}\text { Champ culturel } \\
\text { par rapport à l'ensemble } \\
\text { des secteurs marchands } \\
(e n \%)\end{array}$ \\
\hline Nombre d'unités légales & 156579 & 3324331 & 4,7 \\
\hline Effectifs salariés en équivalent temps plein & 280984 & 12259209 & 2,3 \\
\hline Effectifs salariés au 31 décembre & 341291 & 15053110 & 2,3 \\
\hline Chiffre d'affaires hors taxes (millions d'euros) & 70814 & 3597967 & 2,0 \\
\hline Valeur ajoutée - y compris autres produits & & & \\
\hline et autres charges (millions d'euros) & 28656 & 1033389 & 2,8 \\
\hline Excédent brut d'exploitation (millions d'euros) & 8369 & 281357 & 3,0 \\
\hline Résultat courant avant impôts (millions d'euros) & 3946 & 330827 & 1,2 \\
\hline
\end{tabular}

Source : Insee, Ésane 2010/DEPS, Ministère de la Culture et de la Communication, 2012

d'affaires (CA) général du champ culturel. Les éditions de revues, de journaux et de livres ainsi que la production télévisuelle sont proches en termes de contribution au CA et à la vA (respectivement $7 \%$ et $9 \%$ ). Tous les autres secteurs concourent pour moins de $5 \%$ à l'ensemble du champ culturel marchand.

\section{Une population de petites structures dynamiques}

Les petites structures de moins de 10 salariés forment l'essentiel du tissu économique des secteurs culturels marchands (graphique 1) : l'étude des secteurs culturels à un niveau plus agrégé de la nomenclature NAF confirme cette caractéristique pour les six niveaux culturels ${ }^{3}$ avec des taux de $63 \%$ à $99 \%$ de secteurs constitués d'entreprises de 0 à 9 salariés en équivalent temps plein (ETP).

On observe cependant des variations et trois grands ensembles se dessinent : le premier est formé de secteurs dans lesquels l'essentiel des effectifs et des résultats d'exploitation sont pris en charge par de petites entreprises. Ainsi la quasi-totalité des entreprises de design comptent moins de 10 salariés et réalisent plus des deux tiers du chiffre d'affaires et de la valeur ajoutée. Les petites entreprises, très majoritaires dans les activités créatives, artistiques et de spectacle, réalisent aussi l'essentiel des résultats pour plus de la moitié du chiffre d'affaires et de la valeur ajoutée. Dans ces deux groupes, les entreprises moyennes entre 20 à 249 salariés ETP réalisent le tiers des activités économiques.

Un deuxième ensemble est composé d'un grand nombre de petites entreprises de moins de 10 salariés ETP qui réalisent de $30 \%$ à $50 \%$ du CA et de la vA. Parallèlement coexiste un petit nombre d'entreprises moyennes à grandes. Dans les activités de production et de distribution cinématographiques et télévisuelles ainsi que dans l'édition vidéo, les entreprises de 10 à 19 salariés ETP représentent moins de $3 \%$ du total et réalisent $13 \%$ du CA et de la vA. Celles de 20 à 249 salariés ETP représentent aussi environ $3 \%$ en nombre mais réalisent jusqu'à $51 \%$ du CA et $44 \%$ de la vA.

Dans la musique enregistrée, l'importance économique des très grandes entreprises de 250 salariés ETP est établie : bien que représentant moins de $1 \%$ du total des unités du secteur, elles réalisent $27 \%$ du CA et $23 \%$ de la vA. Dans ce secteur, les petites entreprises de moins de 10 salariés et les entreprises de 20 à 249 salariés réalisent environ les deux tiers du chiffre d'affaires (34\% pour les moins de 10 salariés et $36 \%$ pour les 20 à 249).

Enfin un troisième ensemble atteste d'une concentration encore plus marquée des très grandes entreprises : il réunit les secteurs culturels dans lesquels plus de la moitié et jusqu'à la presque totalité des résultats sont réalisés par un très faible nombre de grandes unités de plus de 250 salariés ETP (moins de $4 \%$ en nombre d'unités du secteur). Il s'agit des secteurs audiovisuels de la radio et de la télévision qui concentrent $53 \%$ à $82 \%$ du CA et $65 \%$ à $85 \%$ de la VA. À l'opposé, $80 \%$ des petites entreprises de moins de 10 salariés de la radio ne génèrent que $6 \%$ de la va et $63 \%$ des petites entreprises de moins de 10 salariés de la télévision produisent une valeur ajoutée négative qui s'explique en partie par des charges élevées, en particulier de sous-traitance.

\section{Avec 6 milliards d'euros de chiffre d'affaires réalisés à l'export, des activités économiques qui s'exportent peu, à l'exception du jeu vidéo}

Avec un chiffre d'affaires total à l'export d'environ 6 milliards d'euros, la proportion des ventes à l'export de l'ensemble des secteurs culturels marchands apparaît faible (8\%) en 2010, comparée au taux moyen de l'ensemble des secteurs marchands $(15 \%)$. Cependant ce taux peut être

3. Seuls 6 niveaux « groupes » de la NAF Rév.2, 2008 sont retenus pour la ventilation par taille d'effectifs car seuls ces groupes sont entièrement culturels, c'est-à-dire composés uniquement d'activités culturelles. Il s'agit de: 59.1 - Activités cinématographiques, vidéo et de télévision; 59.2 - Enregistrement sonore et édition musicale ; 60.1 - Édition et diffusion de programmes radio ; 60.2 - Programmation de télévision et télédiffusion ; 74.1 - Activités spécialisées de design; 90.0 - Activités créatives, artistiques et de spectacle. 


\begin{tabular}{|c|c|c|c|c|c|c|}
\hline \multirow[t]{2}{*}{$\begin{array}{l}\text { Domaine } \\
\text { culturel }\end{array}$} & \multirow[t]{2}{*}{ Libellé } & \multirow[t]{2}{*}{$\begin{array}{l}\text { Code NAF } \\
2008\end{array}$} & \multirow[t]{2}{*}{$\begin{array}{l}\text { Nombre } \\
\text { d'unités } \\
\text { légales }\end{array}$} & \multirow[t]{2}{*}{$\begin{array}{c}\text { Effectifs } \\
\text { salariés } \\
\text { en } \\
\text { équivalent } \\
\text { temps plein }\end{array}$} & $\begin{array}{c}\text { Chiffre } \\
\text { d'affaires } \\
\text { hors } \\
\text { taxes }\end{array}$ & $\begin{array}{l}\text { Valeur ajoutée } \\
\text { y compris } \\
\text { autres } \\
\text { produits } \\
\text { et autres } \\
\text { charges }\end{array}$ \\
\hline & & & & & $\begin{array}{l}\text { (millions } \\
\text { d'euros) }\end{array}$ & $\begin{array}{l}\text { (millions } \\
\text { d'euros) }\end{array}$ \\
\hline \multicolumn{7}{|c|}{ Patrimoine } \\
\hline & Gestion des bibliothèques et des archives & $91.01 Z$ & S & S & S & S \\
\hline & Gestion des musées & $91.02 Z$ & $S$ & S & 42 & 14 \\
\hline & $\begin{array}{l}\text { Gestion des sites et monuments historiques } \\
\text { et des attractions touristiques similaires }\end{array}$ & $91.03 Z$ & 323 & 2042 & 230 & 110 \\
\hline \multicolumn{7}{|c|}{ Livre et presse } \\
\hline & Édition de livres & $58.11 Z$ & 4193 & 12820 & 5942 & 1504 \\
\hline & Édition de journaux & $58.13 Z$ & 809 & 27279 & 5183 & 1797 \\
\hline & Édition de revues et périodiques & $58.14 Z$ & 2433 & 24042 & 6433 & 2187 \\
\hline & Agences de presse & $63.91 Z$ & 2025 & 4428 & 835 & 513 \\
\hline \multicolumn{7}{|c|}{ Arts visuels } \\
\hline & $\begin{array}{l}\text { Création artistique relevant } \\
\text { des arts plastiques }\end{array}$ & $90.03 \mathrm{~A}$ & 6441 & S & S & S \\
\hline & Autre création artistique & $90.03 \mathrm{~B}$ & 13819 & S & S & S \\
\hline & Activités photographiques & $74.20 Z(p)$ & 11013 & 5911 & 1217 & 500 \\
\hline & Activités spécialisées de design & $74.10 Z$ & 20024 & 6584 & 1693 & 724 \\
\hline \multicolumn{7}{|c|}{ Architecture } \\
\hline & Activités d'architecture & $71.11 Z$ & 31646 & 32268 & 7320 & 4135 \\
\hline \multicolumn{7}{|c|}{ Spectacle vivant } \\
\hline & Arts du spectacle vivant & $90.01 Z$ & 11097 & 8090 & 1721 & 574 \\
\hline & Activités de soutien au spectacle vivant & $90.02 Z$ & 5922 & 8522 & 1759 & 694 \\
\hline & Gestion de salles de spectacles & $90.04 Z$ & 514 & 3739 & 959 & 183 \\
\hline \multicolumn{7}{|c|}{ Audiovisuel/multimédia } \\
\hline & $\begin{array}{l}\text { Production de films et de programmes } \\
\text { pour la télévision }\end{array}$ & $59.11 \mathrm{~A}$ & 2690 & 9659 & 2420 & 2084 \\
\hline & $\begin{array}{l}\text { Production de films institutionnels } \\
\text { et publicitaires }\end{array}$ & 59.11B & 4368 & 5058 & 1316 & 531 \\
\hline & Production de films pour le cinéma & $59.11 \mathrm{C}$ & 2114 & 4901 & 1929 & 1402 \\
\hline & $\begin{array}{l}\text { Postproduction de films cinématographiques, } \\
\text { de vidéo et de programmes de télévision }\end{array}$ & $59.12 Z$ & 3913 & 10230 & 2027 & 996 \\
\hline & Distribution de films cinématographiques & $59.13 \mathrm{~A}$ & 498 & 1841 & 1803 & 377 \\
\hline & Édition et distribution vidéo & $59.13 B$ & 786 & 1361 & 783 & 168 \\
\hline & Projection de films cinématographiques & $59.14 Z$ & 863 & 6746 & 1385 & 443 \\
\hline & Édition de jeux électroniques & $58.21 Z$ & 189 & 1811 & 1197 & 385 \\
\hline & Édition d'enregistrements sonores & $59.20 Z$ & 3532 & 3575 & 1182 & 428 \\
\hline & Édition et diffusion de programmes radio & $60.10 Z$ & 362 & 9044 & 1375 & 694 \\
\hline & Édition de chaînes généralistes & $60.20 \mathrm{~A}$ & 26 & 14622 & 7691 & 3139 \\
\hline & Édition de chaînes thématiques & $60.20 \mathrm{~B}$ & 154 & 4533 & 1664 & 231 \\
\hline \multicolumn{7}{|l|}{ Publicité } \\
\hline & Activités des agences de publicité & $73.11 Z(p)$ & 18254 & 70853 & 12462 & 4712 \\
\hline \multicolumn{7}{|c|}{ Éducation/formation } \\
\hline & Enseignement culturel & $85.52 Z$ & 8571 & 1025 & 247 & 134 \\
\hline \multicolumn{3}{|c|}{ Total secteurs culturels marchands } & 156579 & 280984 & 70814 & 28656 \\
\hline \multicolumn{3}{|c|}{ Tous secteurs des services marchands (hors agriculture) } & 3324331 & 12259209 & 3597967 & 1033389 \\
\hline
\end{tabular}


Graphique 1 - Répartition par taille d'effectifs de 6 groupes culturels :

nombre d'unités, chiffre d'affaires et valeur ajoutée en 2010

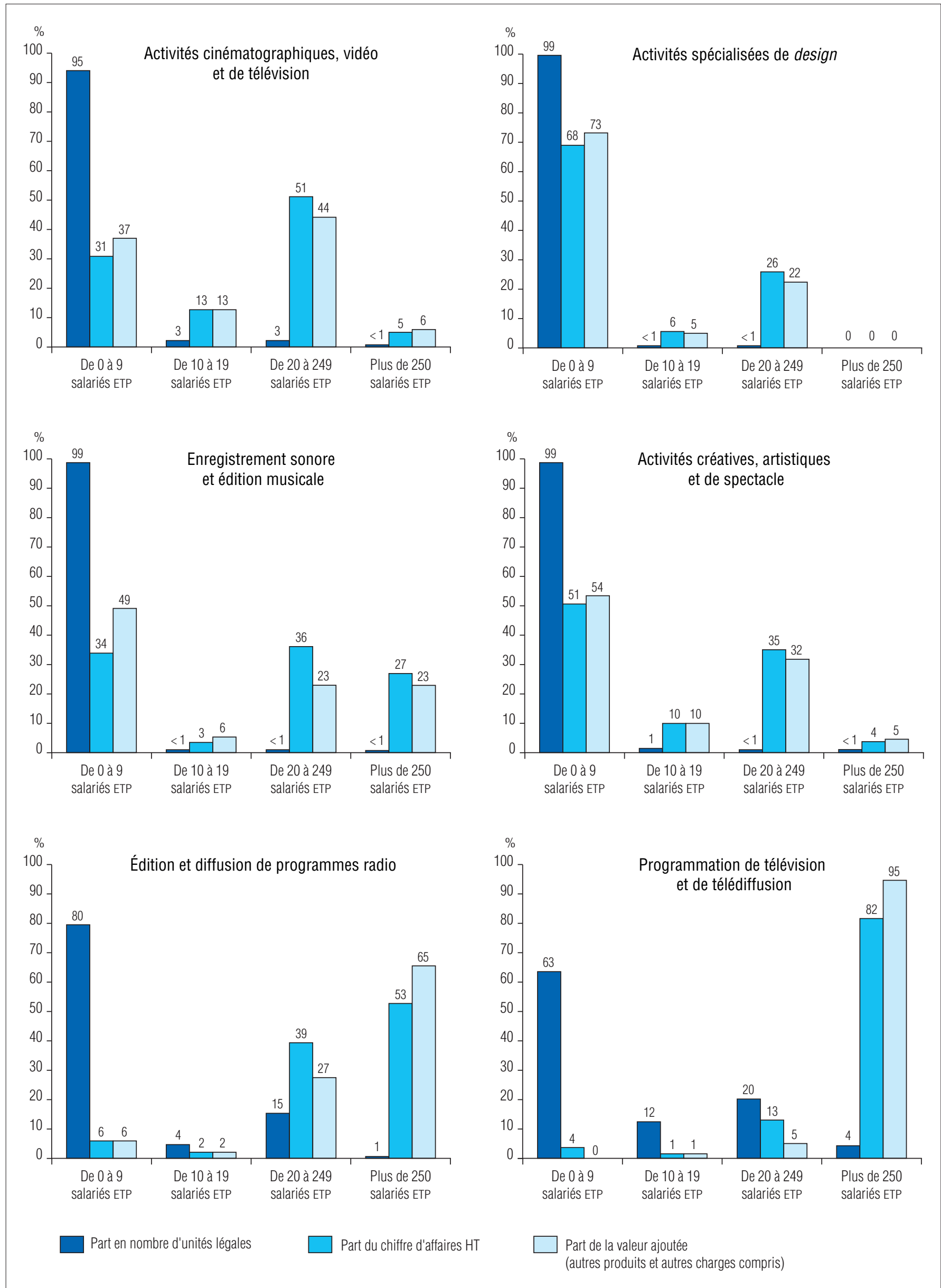

Source : Insee, Ésane 2010/DEPS, Ministère de la Culture et de la Communication, 2012 


\begin{tabular}{|c|c|c|c|c|}
\hline & \multicolumn{4}{|c|}{ Nombre de salariés en ETP } \\
\hline & De 0 à 9 & De 10 à 19 & De 20 à 249 & Plus de 250 \\
\hline Activités cinématographiques, vidéo et de télévision & 23 & 18 & 55 & 5 \\
\hline Enregistrement sonore et édition musicale & 20 & 6 & 31 & 43 \\
\hline Édition et diffusion de programmes radio & 28 & 0 & 69 & 3 \\
\hline Programmation de télévision et télédiffusion & 2 & 0 & 17 & 81 \\
\hline Activités spécialisées de design & 29 & 8 & 63 & 0 \\
\hline Activités créatives, artistiques et de spectacle & 41 & 10 & 46 & 2 \\
\hline
\end{tabular}

rapproché du taux moyen des secteurs de l'information et de la communication (9\%), et il est bien supérieur au taux moyen de l'ensemble des autres activités de services (2\%).

De plus, de fortes différences s'observent selon les secteurs : tandis que les entreprises d'édition de jeux électroniques réalisent plus de $50 \%$ de leur chiffre d'affaires à l'étranger, certains secteurs dont les activités sont traditionnellement proposées sur le marché national tels les musées, les salles de cinéma, l'enseignement culturel, ont des taux d'exportation nuls. Entre ces deux pôles, les secteurs culturels marchands ont des comportements d'exportation différents, avec un taux déterminant autour de $20 \%$

Tableau 4- Taux de marge des secteurs culturels en 2010

\begin{tabular}{|c|c|c|c|c|c|c|c|}
\hline $\begin{array}{l}\text { Code NAF } \\
2008\end{array}$ & Libellé du secteur d'activité & $\begin{array}{l}\text { Valeur } \\
\text { ajoutée }\end{array}$ & $\begin{array}{c}\stackrel{+}{\text { Subventions }} \\
\text { d'exploitation }\end{array}$ & $\begin{array}{c}\text { - } \\
\text { Impôts, } \\
\text { taxes } \\
\text { et versements } \\
\text { assimilés }\end{array}$ & $\begin{array}{c}- \\
\text { Frais } \\
\text { de } \\
\text { personnel }\end{array}$ & $\begin{array}{c}= \\
\text { EBE }\end{array}$ & $\begin{array}{c}\text { Taux } \\
\text { de marge } \\
\text { (en \%) }\end{array}$ \\
\hline $58.11 Z$ & Édition de livres & 1504 & 11 & 69 & 878 & 569 & 38 \\
\hline $58.13 Z$ & Édition de journaux & 1797 & 87 & 74 & 1830 & -20 & -1 \\
\hline $58.14 Z$ & Édition de revues et périodiques & 2187 & 9 & 71 & 1698 & 426 & 20 \\
\hline $58.21 Z$ & Édition de jeux électroniques & 385 & 1 & 7 & 117 & 262 & 68 \\
\hline $59.11 \mathrm{~A}$ & $\begin{array}{l}\text { Production de films et de programmes } \\
\text { pour la télévision }\end{array}$ & 2084 & 99 & 43 & 1006 & 1134 & 54 \\
\hline $59.11 \mathrm{~B}$ & Production de films institutionnels et publicitaires & 531 & 5 & 16 & 438 & 82 & 15 \\
\hline $59.11 C$ & Production de films pour le cinéma & 1402 & 93 & 27 & 648 & 820 & 59 \\
\hline $59.12 Z$ & $\begin{array}{l}\text { Postproduction de films cinématographiques, } \\
\text { de vidéo et de programmes de télévision }\end{array}$ & 996 & 7 & 43 & 809 & 151 & 15 \\
\hline $59.13 \mathrm{~A}$ & Distribution de films cinématographiques & 377 & 14 & 17 & 132 & 243 & 64 \\
\hline $59.13 B$ & Édition et distribution vidéo & 168 & 2 & 9 & 96 & 64 & 38 \\
\hline $59.14 Z$ & Projection de films cinématographiques & 443 & 29 & 51 & 257 & 164 & 37 \\
\hline $59.20 Z$ & Enregistrement sonore et édition musicale & 428 & 9 & 20 & 317 & 100 & 23 \\
\hline $60.10 Z$ & Édition et diffusion de programmes radio & 694 & 38 & 38 & 635 & 59 & 8 \\
\hline $60.20 \mathrm{~A}$ & Édition de chaînes généralistes & 3139 & 198 & 472 & 1262 & 1602 & 51 \\
\hline $60.20 \mathrm{~B}$ & Édition de chaînes thématiques & 231 & 214 & 34 & 365 & 46 & 20 \\
\hline $63.91 Z$ & Activités des agences de presse & 513 & 5 & 13 & 429 & 76 & 15 \\
\hline $71.11 Z$ & Activités d'architecture & 4135 & 19 & 163 & 2623 & 1368 & 33 \\
\hline $73.11 Z(p)$ & Activités des agences de publicité & 4712 & 10 & 216 & 3954 & 552 & 12 \\
\hline $74.10 Z$ & Activités spécialisées de design & 724 & 4 & 31 & 489 & 208 & 29 \\
\hline $74.20 Z$ (p) & Activités photographiques & 500 & 2 & 23 & 330 & 148 & 30 \\
\hline $85.52 Z$ & Enseignement culturel & 134 & 5 & 7 & 78 & 53 & 40 \\
\hline $90.01 Z$ & Arts du spectacle vivant & 574 & 152 & 38 & 545 & 144 & 25 \\
\hline $90.02 Z$ & Activités de soutien au spectacle vivant & 694 & 13 & 26 & 607 & 73 & 11 \\
\hline $90.04 Z$ & Gestion de salles de spectacles & 183 & 86 & 24 & 230 & 17 & 9 \\
\hline $91.02 Z$ & Gestion des musées & 14 & 5 & 1 & 15 & 3 & 22 \\
\hline $91.03 Z$ & $\begin{array}{l}\text { Gestion des sites et monuments historiques } \\
\text { et des attractions touristiques similaires }\end{array}$ & 110 & 19 & 6 & 98 & 26 & 23 \\
\hline Total & Moyenne secteurs culturels marchands & 28656 & 1137 & 1541 & 19883 & 8369 & 29 \\
\hline
\end{tabular}


pour les entreprises d'édition de chaînes thématiques, les agences de presse, la musique enregistrée, la distribution de films cinématographiques et le design. La production cinématographique et de films publicitaires, l'édition de livres, de périodiques et de vidéos, les activités créatives et techniques de spectacles vivants réalisent environ $10 \%$ de leur chiffre d'affaires à l'exportation. Enfin un dernier groupe de secteurs culturels comprenant l'architecture, la photographie, les activités de la radio, la gestion de salles de spectacles ou encore les chaînes généralistes réalisent moins de $5 \%$ de leur chiffre d'affaires en exportant leurs activités.

Il est intéressant de noter la position favorable à l'exportation des petites structures, quelle que soit leur activité (tableau 3) : à l'exception de la programmation télévisuelle, la proportion des unités de moins de 10 salariés à se tourner vers le commerce extérieur atteint $20 \%$ à $40 \%$ de l'ensemble des entreprises du secteur concerné. Avec les entreprises de 20 à 249 salariés ETP, elles sont les plus dynamiques sur le marché extérieur. Le secteur de la musique enregistrée (43\% du chiffre d'affaires réalisé à l'export) et celui de la programmation télévisuelle (81\% à l'export) sont fortement engagés sur le marché international grâce à l'activité des plus grandes entreprises, celles de plus de 250 salariés.

\section{DES PERFORMANCES D'EXPLOITATION CONTRASTÉES SELON LES SECTEURS}

Les frais de personnel (salaires et charges sociales) constituent une part conséquente de la différence entre la valeur ajoutée et l'excédent brut d'exploitation. Ils sont plus ou moins élevés selon les secteurs et leur incidence sur la rentabilité de l'exploitation courante peut être importante (tableau 4) : l'édition de journaux, par exemple, est le seul secteur culturel où l'insuffisance d'exploitation se traduit par un solde négatif en 2010, ce qui s'explique par des frais de personnel élevés non compensés par des subventions d'exploitation.

Le taux moyen de rémunération du travail (soit la part de la valeur ajoutée consacrée aux frais de personnel) dans l'ensemble des secteurs marchands (hors agriculture) est de $66 \%$ (tableau 6), il est très légèrement supérieur pour les seuls secteurs culturels (69\%). Dans le cas de l'édition de journaux, ce taux atteint $102 \%$ (tableau 5) : la valeur ajoutée dégagée ne suffit pas à couvrir les frais de personnel qui lui sont supérieurs, ce qui grève largement la richesse produite. Trois autres secteurs affichent des frais de personnel égaux ou supérieurs à leur valeur ajoutée: les musées

Tableau 5 - Répartition de la valeur ajoutée en 2010

\begin{tabular}{|c|c|c|c|c|}
\hline $\begin{array}{l}\text { Code NAF } \\
2008\end{array}$ & Libellé du secteur d'activité & $\begin{array}{l}\text { subventions } \\
\text { tion par rapport } \\
\text { leur ajoutée }\end{array}$ & $\begin{array}{l}\text { Part des impôts, } \\
\text { taxes par rapport } \\
\text { à la valeur ajoutée }\end{array}$ & $\begin{array}{l}\text { Part des frais de } \\
\text { personnel par rapport } \\
\text { à la valeur ajoutée }\end{array}$ \\
\hline $58.11 Z$ & Édition de livres & 1 & 5 & 58 \\
\hline $58.13 Z$ & Édition de journaux & 5 & 4 & 102 \\
\hline $58.14 Z$ & Édition de revues et périodiques & 0 & 3 & 78 \\
\hline $58.21 Z$ & Édition de jeux électroniques & 0 & 2 & 30 \\
\hline $59.11 \mathrm{~A}$ & Production de films et de programmes pour la télévision & 5 & 2 & 48 \\
\hline $59.11 \mathrm{~B}$ & Production de films institutionnels et publicitaires & 1 & 3 & 82 \\
\hline $59.11 \mathrm{C}$ & Production de films pour le cinéma & 7 & 2 & 46 \\
\hline $59.12 Z$ & $\begin{array}{l}\text { Postproduction de films cinématographiques, } \\
\text { de vidéo et de programmes de télévision }\end{array}$ & 1 & 4 & 81 \\
\hline $59.13 \mathrm{~A}$ & Distribution de films cinématographiques & 4 & 5 & 35 \\
\hline 59.13B & Édition et distribution vidéo & 1 & 5 & 57 \\
\hline $59.14 Z$ & Projection de films cinématographiques & 7 & 12 & 58 \\
\hline $59.20 Z$ & Enregistrement sonore et édition musicale & 2 & 5 & 74 \\
\hline $60.10 Z$ & Édition et diffusion de programmes radio & 5 & 5 & 92 \\
\hline $60.20 \mathrm{~A}$ & Édition de chaînes généralistes & 6 & 15 & 40 \\
\hline $60.20 \mathrm{~B}$ & Édition de chaînes thématiques & $92^{*}$ & 15 & 158 \\
\hline $63.91 Z$ & Activités des agences de presse & 1 & 3 & 84 \\
\hline $71.11 Z$ & Activités d'architecture & 0 & 4 & 63 \\
\hline $73.11 Z$ & Activités des agences de publicité & 0 & 5 & 84 \\
\hline $74.10 Z$ & Activités spécialisées de design & 1 & 4 & 68 \\
\hline $74.20 Z$ & Activités photographiques & 0 & 5 & 66 \\
\hline $85.52 Z$ & Enseignement culturel & 4 & 5 & 59 \\
\hline $90.01 Z$ & Arts du spectacle vivant & 26 & 7 & 95 \\
\hline $90.02 Z$ & Activités de soutien au spectacle vivant & 2 & 4 & 87 \\
\hline $90.04 Z$ & Gestion de salles de spectacles & 47 & 13 & 125 \\
\hline $91.02 Z$ & Gestion des musées & 38 & 6 & 111 \\
\hline $91.03 Z$ & $\begin{array}{l}\text { Gestion des sites et monuments historiques } \\
\text { et des attractions touristiques similaires }\end{array}$ & 18 & 6 & 88 \\
\hline
\end{tabular}


(111\%), les salles de spectacles (125\%) et l'édition de chaînes thématiques (158\%). Pour ces secteurs, en revanche, les frais de personnel élevés sont compensés par des subventions d'exploitation, elles aussi élevées (respectivement de $38 \%, 47 \%$ et $92 \%$ de la valeur ajoutée) et très supérieures à la moyenne des secteurs culturels (4\%).

Le profit dégagé par les secteurs est lié à l'équilibre entre rémunération du travail (elle-même liée à la politique d'emploi et au niveau de qualification du personnel) et impôts sur la production, auxquels s'ajoutent l'ensemble des transferts courants des administrations publiques et les choix d'investissement. Les subventions d'exploitation, au sens de la comptabilité, correspondent à tout type de transferts courants versés par les administrations publiques. Ces subventions d'exploitation sont destinées à financer le fonctionnement de l'entreprise et sont accordées pour différentes destinations : elles peuvent, par exemple, être demandées pour faciliter l'emploi de certaines catégories d'actifs (primes à l'emploi de chômeurs âgés de 45 ans et plus, Rmistes, etc.) ou encore en tant que soutien pour l'acquisition de matériels radiophoniques. Ces transferts courants des administrations permettent aux entreprises de compenser l'insuffisance de certains postes de leur exploitation régulière en équilibrant le budget et en générant ainsi une marge brute positive.

En 2010, la part des subventions d'exploitation rapportée au total de la valeur ajoutée est de $2 \%$ pour l'ensemble des secteurs marchands non agricoles (tableau 6) ; dans les secteurs culturels marchands, ce taux moyen est proche, supérieur de 2 points seulement (4\%). Comparés à cette moyenne, cinq secteurs bénéficient de subventions considérables sur les produits ou sur l'exploitation (tableau 5) : l'édition de chaînes thématiques (92\% de la VA), la gestion de salles de spectacles (47\%), les musées (38\%), les arts du spectacle vivant (26\%), les sites et monuments historiques $(18 \%)$. À l'exception de ces cinq secteurs, les taux de subventions d'exploitation des secteurs culturels sont comparables à la moyenne des secteurs culturels marchands ( $4 \%$ ), avec des variations qui n'excèdent pas de 3 points la moyenne (plus ou moins). Le volume des subventions d'exploitation est anecdotique par rapport à la valeur ajoutée dans le cas de l'architecture, la photographie, l'édition de jeux électroniques et de revues, ainsi que les agences de publicité (tableau 5).

Dans les analyses futures permises par la définition récente du champ des activités culturelles, il sera intéressant de retenir ces indicateurs de répartition de la valeur ajoutée pour mesurer la stabilité ou l'évolution des salaires, des taxes et des subventions d'exploitation sur plusieurs années.

Le taux de marge, soit le rapport de l'excédent brut d'exploitation sur la valeur ajoutée, mesure la rentabilité en précisant la part de la valeur créée par l'entreprise qui sert à rémunérer les apporteurs de capitaux et à renouveler le capital investi (graphique 2). Les taux de marge élevés sont plutôt le fait de secteurs qui ont une importante activité capitalistique, tels les secteurs des télécommunications par satellite ( $86 \%$ en 2010) ou les activités de commerce des agents immobiliers (71\%). À l'inverse, les taux de marge faibles dénotent d'un relatif faible emploi du capital par rapport au travail.

\section{Tableau 6-Taux de marge des secteurs d'activités culturels en 2010}

\begin{tabular}{|lccccc}
\hline & $\begin{array}{c}\text { Taux de marge } \\
\text { (EBE par rapport } \\
\text { à la valeur ajoutée) }\end{array}$ & $\begin{array}{c}\text { Part des subventions } \\
\text { d'exploitation par rapport } \\
\text { à la valeur ajoutée }\end{array}$ & $\begin{array}{c}\text { Part des impôts, } \\
\text { taxes par rapport } \\
\text { à la valeur ajoutée }\end{array}$ & $\begin{array}{c}\text { Part des frais de } \\
\text { personnel par rapport } \\
\text { à la valeur ajoutée }\end{array}$ & $\begin{array}{c}\text { Taux de } \\
\text { profitabilité } \\
\text { (RNc/ca) }\end{array}$ \\
$\begin{array}{l}\text { Secteurs culturels marchands } \\
\begin{array}{c}\text { Ensemble des secteurs } \\
\text { des services marchands }\end{array}\end{array}$ & 29 & 4 & 5 & 69 \\
8
\end{tabular}

\section{Graphique 2-Taux de marge des secteurs d'activités culturels en 2010}

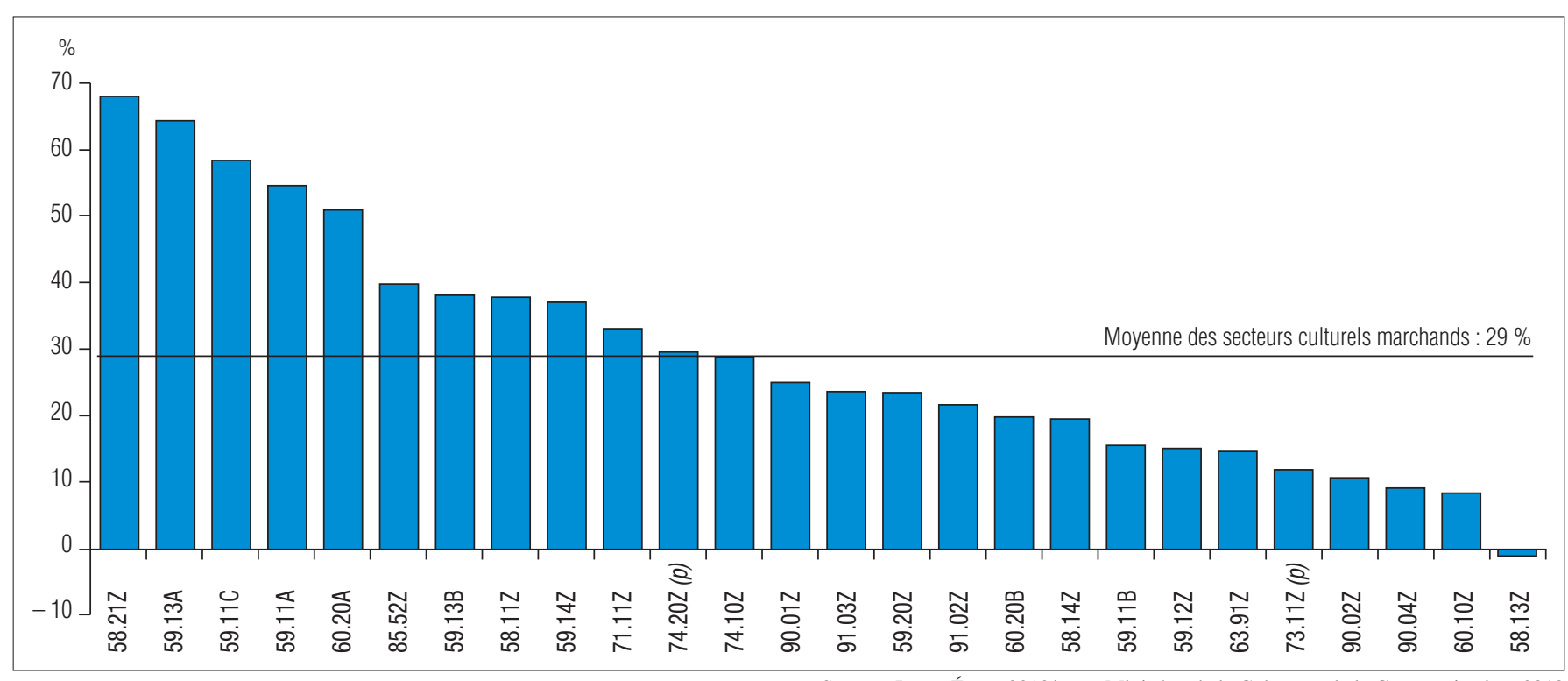

Source : Insee, Ésane 2010/DEPS, Ministère de la Culture et de la Communication, 2012 
Le taux de marge moyen de l'ensemble des services marchands est de $29 \%$ en 2010 : onze secteurs culturels ont un taux de marge supérieur à cette moyenne. Le taux de marge le plus élevé est celui des activités d'édition de jeux électroniques (68\%). Les activités de distribution de films, de production cinématographique et télévisuelle et l'édition de chaînes généralistes suivent avec des taux de $64 \%$ à $51 \%$. L'enseignement culturel, l'édition vidéo et l'édition de livres, la projection de films cinématographiques ont des taux de marge brute d'environ $40 \%$. Les activités techniques d'architecture, de photographie et de design réalisent des taux proches de $30 \%$. La création en spectacle vivant, la gestion de musées et monuments, la musique enregistrée, l'édition de chaines thématiques et de journaux suivent avec des taux d'environ $20 \%$. Les taux les plus faibles de $15 \%$ ou moins concernent la production de films publicitaires, la publicité et les agences de presse, la postproduction audiovisuelle, les activités techniques et de salles de spectacle vivant et les activités de la radio.

\section{RÉSULTATS COMPTABLES POSITIFS ET PROFITABILITÉ NE SONT PAS TOUJOURS SYNONYMES}

Le résultat courant avant impôts ne tient pas compte des événements qui pourraient perturber l'activité des entreprises alors même qu'ils ne lui seraient pas directement liés (par exemple des opérations de gestion non récurrentes comme la vente d'actifs ou de matériels) : il mesure donc l'impact direct de l'endettement de l'entreprise sur son activité et sa rentabilité économique.

Il est ainsi intéressant de constater que, parallèlement à des résultats de production positifs et à un taux de marge élevé, l'édition de jeux électroniques affiche un résultat net courant avant impôts négatif équivalent à - 102 millions d'euros en 2010. En effet, même si la production totale de biens et services est élevée dans ce secteur, son niveau d'endettement, constaté dans ses dotations d'exploitation

Tableau 7-Profitabilité des secteurs d'activités en 2010

\begin{tabular}{|c|c|c|c|c|c|}
\hline $\begin{array}{l}\text { Secteur } \\
\text { d'activité }\end{array}$ & Activité & $\begin{array}{l}\text { Résultat courant } \\
\text { avant impôts }\end{array}$ & $\begin{array}{l}\text { Chiffre d'affaires } \\
\text { hors taxes }\end{array}$ & $\begin{array}{l}\text { Résultat net } \\
\text { comptable }\end{array}$ & $\begin{array}{l}\text { Profitabilité } \\
\quad \text { (en \%) }\end{array}$ \\
\hline $90.03 \mathrm{~A}$ & Création artistique relevant des arts plastiques & $S$ & S & S & S \\
\hline $90.03 \mathrm{~B}$ & Autre création artistique & S & S & S & S \\
\hline $91.01 Z$ & Gestion des bibliothèques et des archives & S & S & S & S \\
\hline $85.52 Z$ & Enseignement culturel & 41 & 247 & 40 & 16 \\
\hline $71.11 Z$ & Activités d'architecture & 1289 & 7320 & 1156 & 16 \\
\hline $74.10 Z$ & Activités spécialisées de design & 192 & 1693 & 171 & 10 \\
\hline $58.11 Z$ & Édition de livres & 552 & 5942 & 548 & 9 \\
\hline $59.20 Z$ & Enregistrement sonore et édition musicale & 212 & 1182 & 95 & 8 \\
\hline $59.14 Z$ & Projection de films cinématographiques & 99 & 1385 & 102 & 7 \\
\hline $74.20 Z$ (p) & Activités photographiques & 85 & 1217 & 88 & 7 \\
\hline $59.11 \mathrm{~A}$ & Production de films et de programmes pour la télévision & 194 & 2420 & 169 & 7 \\
\hline $59.11 \mathrm{C}$ & Production de films pour le cinéma & -56 & 1929 & 102 & 5 \\
\hline $90.01 Z$ & Arts du spectacle vivant & 88 & 1721 & 78 & 5 \\
\hline $73.11 Z(p)$ & Activités des agences de publicité & 562 & 12462 & 396 & 3 \\
\hline $59.13 \mathrm{~B}$ & Édition et distribution vidéo & 29 & 783 & 24 & 3 \\
\hline $58.14 Z$ & Édition de revues et périodiques & 383 & 6433 & 190 & 3 \\
\hline $90.04 Z$ & Gestion de salles de spectacles & 17 & 959 & 27 & 3 \\
\hline $59.11 \mathrm{~B}$ & Production de films institutionnels et publicitaires & 51 & 1316 & 34 & 3 \\
\hline $91.02 Z$ & Gestion des musées & 1 & 42 & 1 & 1 \\
\hline $90.02 Z$ & Activités de soutien au spectacle vivant & 36 & 1759 & 24 & 1 \\
\hline $60.20 \mathrm{~A}$ & Édition de chaînes généralistes & 311 & 7691 & 89 & 1 \\
\hline $91.03 Z$ & $\begin{array}{l}\text { Gestion des sites et monuments historiques } \\
\text { et des attractions touristiques similaires }\end{array}$ & 6 & 230 & 2 & 1 \\
\hline $59.12 Z$ & $\begin{array}{l}\text { Postproduction de films cinématographiques, } \\
\text { de vidéo et de programmes de télévision }\end{array}$ & 7 & 2027 & 16 & 1 \\
\hline $63.91 Z$ & Activités des agences de presse & 16 & 835 & 1 & 0 \\
\hline $60.10 Z$ & Édition et diffusion de programmes radio & 10 & 1375 & -2 & 0 \\
\hline $59.13 \mathrm{~A}$ & Distribution de films cinématographiques & -16 & 1803 & -29 & -2 \\
\hline $58.13 Z$ & Édition de journaux & -159 & 5183 & -327 & -6 \\
\hline $60.20 \mathrm{~B}$ & Édition de chaînes thématiques & 99 & 1664 & -124 & -7 \\
\hline $58.21 Z$ & Édition de jeux électroniques & -102 & 1197 & -147 & -12 \\
\hline \multicolumn{2}{|c|}{ Secteurs culturels marchands } & 3946 & 70814 & 2721 & 4 \\
\hline \multicolumn{2}{|c|}{ Tous secteurs des services marchands (hors agriculture) } & 330827 & 3597967 & 285490 & 8 \\
\hline
\end{tabular}


aux provisions et aux amortissements (exemple : amortissement d'un emprunt correspondant au remboursement de capitaux empruntés pour un investissement en matériels) l'est lui aussi et conduit à fortement amputer son résultat courant en 2010. D'autres secteurs sont dans des situations comparables conduisant à des taux de profitabilité ${ }^{4}$ négatifs en 2010 (tableau 7) : il s'agit de l'édition de chaînes thématiques, de l'édition de journaux et de la distribution de films cinématographiques. À l'opposé, les activités du champ culturel marchand dont la profitabilité est la plus élevée en 2010 sont celles de l'architecture (16\%), de l'enseignement culturel (16\%) et du design (10\%). Cinq secteurs culturels marchands ont un taux de profitabilité égal ou supérieur au taux moyen de l'ensemble des services marchands (hors agriculture) qui est égal à $8 \%$.

\section{Définitions}

Unité légale : l'unité légale est une entité juridique de droit public ou privé. Cette entité juridique peut être : 1. une personne morale, dont l'existence est reconnue par la loi indépendamment des personnes ou des institutions qui la possèdent ou qui en sont membres; 2 . une personne physique, qui, en tant qu'indépendant, peut exercer une activité économique.

Effectif salarié en équivalent temps plein : les effectifs mesurés en « équivalent temps plein » (ETP) correspondent au nombre total d'heures travaillées dans l'activité considérée divisé par la moyenne annuelle des heures travaillées dans des emplois à plein-temps. Pour éviter de compter une même personne plusieurs fois, on mesure l'emploi en ETP. L'effectif salarié ETP est l'indicateur utilisé pour déterminer les tailles d'entreprises (et contraintes légales s'y référant), ou pour établir des indicateurs (exemple : rendement de la main-d'œuvre, soit le rapport de la valeur ajoutée à l'effectif salarié ETP).

Effectif salarié au 31 décembre : effectif salarié total inscrit dans l'entreprise au 31 décembre.

Chiffre d'affaires hors taxe (СAHT) : montant hors taxes des affaires réalisées par l'entreprise avec les tiers dans l'exercice de son activité professionnelle. Le chiffre d'affaires est constitué par les ventes de marchandises, de la production des biens et des services (France + exportations et livraisons intracommunautaires).

Valeur ajoutée hors taxe, y compris autres produits et autres charges : différence entre la production globale de l'entreprise (marge commerciale + production de l'exercice) et les consommations de biens et de services en provenance des tiers + autres produits - autres charges. La valeur ajoutée mesure la capacité d'une entreprise à générer de la richesse et donc à se maintenir et se développer. Cette richesse se répartit entre plusieurs partenaires de l'entreprise : les salariés (salaires et charges sociales), l'État (par le biais des impôts et taxes), les banques (charges financières), l'entreprise elle-même (autofinancement : bénéfice net, dotation aux amortissements et aux provisions).

Excédent brut d'exploitation (EBE) : autre solde intermédiaire calculé, il est la différence entre la valeur ajoutée + les subventions d'exploitation - les impôts et taxes (sauf l'impôt sur les sociétés) et les charges de personnel supportés par l'entreprise. L'EBE représente la part de la valeur ajoutée qui revient à l'entreprise et aux apporteurs de capitaux (associés et prêteurs). II indique la ressource générée par la seule exploitation de l'entreprise indépendamment de la politique d'amortissements (dotations) et du mode de financement (charges financières).

Résultat courant avant impôt : il est égal au résultat d'exploitation majoré des produits financiers et minoré des charges financières + opérations en commun (bénéfice attribué) - opérations en commun (perte supportée). II mesure la performance de l'activité économique autant que financière de l'entreprise sans intégrer les opérations exceptionnelles ou fiscales qui modifient les résultats.

Résultat net comptable (RNC) : il mesure les ressources nettes (après prise en compte de la dépréciation du capital) restant à l'entreprise à l'issue de l'exercice : bénéfice ou perte. Il correspond, au sens comptable, à la différence entre les produits et les charges de l'exercice.

4. Le taux de profitabilité rapporte le bénéfice au chiffre d'affaires : c'est le rapport du résultat net comptable sur le chiffre d'affaires hors taxe. C'est une mesure de la performance économique globale et non une mesure de la rentabilité. 


\section{Encadré méthodologique : le champ statistique}

\section{Secteurs}

La nomenclature d'activités française (NAF) est la nomenclature statistique qui fait autorité en France pour répertorier les activités économiques, définir des champs d'enquêtes et produire des résultats sectoriels socio-économiques. Tout en étant liée aux nomenclatures européenne et internationale, afin de faciliter l'organisation des marchés et la comparabilité des analyses, la nomenclature nationale française classifie avec plus de précision certains secteurs comme ceux de la création artistique (différenciation entre la création en arts plastiques et celle des arts littéraires ou de la composition musicale). En considérant le champ théorique des activités culturelles fondé sur les fonctions et domaines culturels, 29 classes d'activités statistiques françaises NAF Rév.2, 2008 ont été sélectionnées :

- le patrimoine est structuré autour des activités de gestion des bibliothèques et des archives (91.01Z), gestion des musées (91.02Z), gestion des sites et monuments historiques et des attractions touristiques similaires (91.03Z);

- le livre et la presse correspondent à l'édition de livres (58.11Z), l'édition de journaux (58.13Z), l'édition de revues et périodiques (58.14Z) et les agences de presse (63.91Z);

- les arts visuels comprennent la création artistique relevant des arts plastiques (90.03A), l'« autre création artistique " (90.03B), les activités photographiques $(74.20 Z p)$ et les activités spécialisées de design (74.10Z) ;

- l'architecture est référencée en activités d'architecture (71.11Z);

- le spectacle vivant se répartit dans les arts du spectacle vivant (90.01Z), les activités de soutien au spectacle vivant (90.02Z) et la gestion de salles de spectacles (90.04Z);

- l'audiovisuel et le multimédia incluent la production de films et de programmes pour la télévision $(59.11 \mathrm{~A})$, la production de films institutionnels et publicitaires (59.11B), la production de films pour le cinéma (59.11C), la postproduction de films cinématographiques, de vidéo et de programmes de télévision (59.12Z), la distribution de films cinématographiques (59.13A), l'édition et distribution vidéo (59.13B), la projection de films cinématographiques (59.14Z), l'édition de jeux électroniques (58.21Z), l'édition d'enregistrements sonores (59.20Z), l'édition et diffusion de programmes radio (60.10Z), l'édition de chaînes généralistes $(60.20 \mathrm{~A})$ et l'édition de chaînes thématiques (60.20B);

- la publicité s'estime à partir des activités des agences de publicité $(73.11 Z p)$

- enfin une activité transversale d'éducation et de formation est repérable à travers le code d'enseignement culturel (85.52Z).

La nomenclature NAF 2008 a permis à certains secteurs culturels d'être reconnus en tant que secteurs d'activités statistiques à part entière, alors qu'ils étaient dilués dans des secteurs plus vastes dans la NAF 2003. Ainsi, les classes d'activités statistiques du design (74.10Z), de l'édition de jeux électroniques (58.21Z) et d'enseignement culturel (85.52Z) ont été spécifiquement créées en 2008. De même, la gestion des musées (91.02Z), la création en arts plastiques $(90.03 \mathrm{~A})$ et le soutien au spectacle vivant $(90.02 \mathrm{Z})$ ont été précisés. Ce changement de nomenclature en 2008 et ces entrées de nouvelles activités dans le champ statistique de la culture ont d'un côté élargi la visibilité et la mesure de ce champ. De l'autre, ils ont conduit à des ruptures de contours: il est donc nécessaire d'utiliser avec précaution les données sectorielles pour lesquelles des évolutions importantes peuvent apparaître.

Par ailleurs, deux activités statistiques, celles des activités photographiques et publicitaires (74.20Z et $73.11 Z$ ), ne sont que partiellement culturelles $(p)$ car elles agrègent activités culturelles et activités techniques non culturelles : ainsi les activités photographiques comprennent non seulement des services de création (activités des photographes indépendants ou en magasin, des agences, des photothèques) ou de diffusion photographique (édition, expositions, marché de l'art) mais aussi les services de laboratoires (façonniers) et les ventes d'équipement connexe et de consommables. Ainsi les laboratoires tels Cewe Colors, Fujifilm, Photobox ou Photomaton réalisent les chiffres d'affaires les plus importants du secteur, ventilés en services photographiques et activités de commerce de détail.

Les données de ces deux activités sont néanmoins diffusées dans ce document au niveau sectoriel ${ }^{1}$ : quantifier la part des activités spécifiquement culturelles, comme par exemple la seule création photographique dans l'ensemble du secteur de la photographie, nécessiterait de disposer de ventilation fine des produits et services. L'exemple de la photographie permet de rendre compte de l'évolution de ce secteur qui subit d'importants changements liés à l'innovation et au numérique (tirages et albums numériques, objets personnalisés, diversification des activités des photographes).

Les activités des agences de publicité sont par ailleurs habituellement étudiées car elles sont rattachées au mode de financement des industries culturelles (audiovisuel, livres et presse) qui représentent la part la plus importante des investissements médias des annonceurs ${ }^{2}$.

L'éducation culturelle est définie dans une classe d'activités nommée " enseignement culturel » et incluse ainsi dans le champ.

À l'inverse, l'artisanat d'art qui n'a pas la cohérence d'un secteur économique formel n'est pas identifié comme activité spécifique et son analyse relève plutôt des métiers et des produits (produits artisanaux versus produits manufacturés).

\section{Source utilisée}

On privilégie les sources d'enquêtes statistiques générales car elles sont garantes de données objectives, comparables et cohérentes dans le temps: on utilise ici les sources statistiques du dispositif Ésane ${ }^{3}$ de l'Insee, l'élaboration des statistiques annuelles d'entreprises qui couvrent les sociétés et entreprises individuelles non financières et non agricoles.

Le dispositif Ésane a la particularité de rapprocher sources administratives (fichiers fiscaux des déclarations annuelles sur les bénéfices et déclarations annuelles de données sociales - DADS) et résultats d'enquêtes (l'enquête sectorielle annuelle - ESA). L'enquête ESA a vocation à mettre à jour l'activité principale des unités légales et à produire des statistiques sectorielles spécifiques sur la ventilation du chiffre d'affaires, les dépenses particulières, les investissements, la clientèle et sur toute donnée non disponible par l'intermédiaire des sources administratives. Les sources administratives de leur côté permettent de compléter le champ sur les secteurs d'activités non couverts par l'enquête ESA et de limiter la charge de réponse à l'enquête des entreprises en utilisant les données déjà disponibles (fiscales et sur l'emploi et les rémunérations).

1. Un secteur d'activités regroupe toutes les entreprises qui ont la même activité principale (au regard de la nomenclature d'activité économique, NAF Rév.2, 2008). Cette activité principale est attribuée à l'entreprise selon un algorithme s'appuyant principalement sur la déclaration du chiffre d'affaires et sur l'activité dont la contribution au CA est la plus importante. À l'inverse les résultats par branches d'activités décrivent l'activité elle-même, que celle-ci soit réalisée à titre principal ou à titre secondaire par l'entreprise. Les résultats par branche incluent donc des entreprises appartenant à différents secteurs.

2. En $2010,28 \%$ des 30,7 milliards d'euros d'investissements des annonceurs ont été consacrés aux médias des industries culturelles - presse, radio, télévision, cinéma. (Source : les Chiffres clés des annonceurs, Union des annonceurs, 2011).

3. Voir Insee, rubrique Définitions et méthodes/Sources et méthodes/Ésane: http://www.insee.fr/fr/methodes/default.asp?page=sources/ope-elaborationstat-an-entreprises.htm 


\section{Champ marchand}

Le champ Ésane comprend les sociétés juridiques considérées comme marchandes dans le répertoire REE-Sirène immatriculant les entreprises et leurs établissements.

En général, une unité est considérée comme marchande lorsque ses coûts de production sont couverts à plus de $50 \%$ par ses ventes. Elle est considérée comme non marchande dans le cas contraire. À ce premier critère s'en ajoutent d'autres, tels la catégorie juridique et l'effectif salarié (sont exclus les associés gérants, certains établissements publics, les administrations, les entreprises sans salarié). Enfin, l'attribution du critère marchand par les comptables nationaux est prise en compte (par exemple, les associations travaillant au service des entreprises sont marchandes).

Les données 2010 illustrent les caractéristiques économiques des unités légales, ce critère juridique incluant personnes morales et personnes physiques (soit les sociétés, les entreprises individuelles, les parties d'administrations publiques et certaines associations).

Le champ s'est élargi au régime de l'auto-entrepreneur, nouvelle-

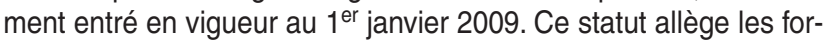
malités de création d'entreprise et repose sur un mode de calcul et de paiement simplifié des cotisations sociales et de l'impôt sur le revenu pour les personnes physiques. L'auto-entrepreneur doit réaliser moins de 80300 euros de chiffre d'affaires annuel pour une activité commerciale et moins de 32100 euros pour les prestations de services et activités libérales.

Avertissement : le champ représenté ici n'étant que le seul champ marchand, certains secteurs d'activité comme ceux du patrimoine, du spectacle vivant ou de l'enseignement affichent des résultats plus faibles en termes de nombre d'unités et d'emplois par rapport au champ complet des acteurs de la culture. Ainsi, le champ total des entreprises culturelles marchandes et non marchandes (hors associations non marchandes sans salarié) est de 164816 unités en 2010, parmi lesquelles $7 \%$ sont des associations employeuses ${ }^{4}$. Dans l'enseignement culturel, les associations représentent $16 \%$ des unités en 2010 ; dans la gestion des musées, bibliothèques et archives, elles représentent $50 \%$ et plus des unités et dans les salles de spectacles, $61 \%$.

\section{Indicateurs retenus}

Exploiter des sources nationales administratives et des enquêtes statistiques permet de disposer de données de qualité autorisant la comparabilité. À ce premier critère de choix s'ajoute celui de la disponibilité des variables quantitatives qui diffère selon les secteurs. C'est pourquoi seules des variables économiques de base sont utilisées afin de décrire les postes principaux des caractéristiques économiques des secteurs culturels et leur part dans l'ensemble de l'économie : nombre d'unités légales, chiffre d'affaires (en tant que production vendue), effectifs en équivalent temps plein, valeur ajoutée et excédent brut d'exploitation.

4. Source : Insee, Répertoire des entreprises et des établissements, Sirène, 2010

\section{RÉSUMÉ}

Les vingt-neuf secteurs statistiques qui forment le champ des entreprises marchandes de la culture regroupent environ 157000 unités en 2010. Ces entreprises culturelles participent pour $5 \%$ du nombre total des entreprises de l'économie marchande française et pour près de $3 \%$ de la valeur ajoutée. Alors que ces secteurs d'activités sont essentiellement formés de petites entreprises de moins de 10 salariés, leurs résultats comptables affichent un poids prépondérant des entreprises moyennes ou grandes. La marge moyenne dégagée par l'ensemble des secteurs culturels est comparable à celle de tous les autres services marchands.

Cependant, ces résultats dévoilent une variété de situations sectorielles, tant au niveau des structures que de leurs résultats commerciaux. Ainsi, les performances économiques des secteurs se traduisent très différemment selon la part des subventions ou celle des frais de personnel sur la valeur ajoutée dégagée.

\section{ABSTRACT}

In 2010, the twenty nine statistical sectors within the field of cultural market businesses covered some 157,000 units. These cultural businesses represent 5\% of the total number of France's market-oriented economy businesses and around $3 \%$ of added value. Whilst these business sectors are essentially composed of small businesses employing fewer than 10 people, their financial results dominate within medium-sized or large businesses. The average margin achieved by the cultural sectors overall is comparable with that of all other market-oriented services.

However, these results reveal a wide range of individual situations, in terms of both structure and financial results. Therefore, the sectors' economic performances are extremely varied depending on the impact of subsidy levels and staffing costs on the added value generated.

\section{Tous les documents publiés par le DEPs sont téléchargeables sur http://www.culturecommunication.gouv.fr/Etudes-et-statistiques et sur www.cairn.info}

Pour recevoir régulièrement les publications du DEPS et pour toute demande d'information: contact.deps@culture.gouv.fr 